\title{
DISCONNECTED GLEASON PARTS
}

\author{
BY JOHN GARNETT ${ }^{1}$
}

Communicated by I. Singer, November 30, 1965

Let $A$ be an algebra of continuous complex valued functions on a compact Hausdorff space $X$. If $A$ is uniformly closed, contains the constant functions and separates the points of $X$, then we call $A$ a function algebra on $X . M(A)$, the space of all multiplicative linear functionals on $A$, is a compact Hausdorff space containing a homeomorphic image of $X$ and $A$ can be viewed as a function algebra on $M(A)$. In [1] Gleason observed that the relation $x \sim y$ if and only if $\|x-y\|_{A^{*}}<2$ is an equivalence relation on $M(A)$. The equivalence classes are called the "parts" of $M(A)$.

Hoffman has asked if parts must be connected. The purpose of this note is to exhibit some disconnected parts.

The author wishes to thank his thesis advisor, Professor Irving Glicksberg, and Professor Alexander Pełczyński for many helpful conversations.

THEOREM 1. There is a function algebra $A$ with two generators such that $M(A)$ contains a part homeomorphic to two copies of the open unit disc.

THEOREM 2. Let $K$ be a compact Hausdorff space. Then there exists a function algebra $A$ such that $M(A)$ contains a part homeomorphic to $K$. Every continuous function on that part is the restriction of a function in $A$.

The last assertion implies that no subset of the part can be given any analytic structure. We will prove Theorem 1 here. The proof of Theorem 2 is more complicated and will be presented elsewhere.

The disc algebra is the algebra of all functions continuous on the closed unit disc $D$ and analytic on the interior. $T^{1}$ denotes the unit circle in the complex plane and $T^{2}$ denotes the torus $T^{1} \times T^{1}$.

Proof of Theorem 1. Pick a function $h_{1}$ in the disc algebra mapping $D$ onto $\{z \in C:|z| \leqq 1,|z-1| \leqq 3 / 4\}$. Let $h_{2}=-h_{1}$. Choose an open $\operatorname{arc} I$ on $T^{1}$ such that $\left|h_{1}\right|=1$ on $I$ and set

$$
\begin{aligned}
X_{j} & =\left\{(z, w) \in T^{2}: z \in I, w=h_{j}(z)\right\} \quad(j=1,2), \\
Y & =\left\{(z, w) \in T^{2}: z \notin I\right\} .
\end{aligned}
$$

1 The author holds a National Science Foundation Fellowship. This research is a part of a doctoral dissertation to be submitted to the University of Washington. 
Then $X=X_{1} \cup X_{2} \cup Y$ is a compact subset of $T^{2}$. Our algebra $A$ is $P(X)$, the uniform closure on $X$ of the polynomials in the coordinate functions $z$ and $w . M(A)$ is the polynomial hull of $X$,

$$
\hat{X}=\left\{p \in C^{2}:|f(p)| \leqq \max _{x \in X}|f(x)|, \text { for every polynomial, } f\right\} .
$$

Set

$$
\begin{aligned}
V & =\{(z, w) \in \hat{X}:|z|<1\}, \\
V_{j} & =\left\{(z, w) \in C^{2}:|z|<1, w=h_{j}(z)\right\} \quad(j=1,2) .
\end{aligned}
$$

We show that $V=V_{1} \cup V_{2}$ and that $V$ is a part of $X$. The proof is in two steps.

(i) $V$ is the disjoint union $V_{1} \cup V_{2}$.

Since $f\left(z, h_{j}(z)\right)$ is in the disc algebra for any polynomial $f$ and $\left\{\left(z, h_{j}(z)\right):|z|=1\right\} \subset X_{j} \cup \hat{Y} \subset \hat{X}$, we have $V_{j} \subset V$. Because $h_{1}$ never vanishes, $V_{1} \cap V_{2}=\varnothing$.

Take $q=\left(z^{0}, w^{0}\right) \in V$ and let $m$ be a representing measure on $X$ for $q$. Let $\mu$ be the measure

$$
\mu=\left(w-h_{1}(z)\right)\left(w-h_{2}(z)\right)\left(z-z^{0}\right) m .
$$

Then $\mu$ is supported by $Y$ and annihilates $A$ because the multiplicative measure $m$ has been multiplied by a function in $A$ vanishing on $X_{1} \cup X_{2} \cup\{q\}$. Hence $\mu$ is orthogonal to $P(Y)$. Now the restricted function $\left(z-z^{0}\right) \mid Y$ is invertible in $P(Y)$ so that the measure

$$
\frac{1}{z-z^{0}} \mu=\left(w-h_{1}(z)\right)\left(w-h_{2}(z)\right) m
$$

annihilates all polynomials. But then

$$
0=\int 1\left(w-h_{1}(z)\right)\left(w-h_{2}(z)\right) d m=\left(w^{0}-h_{1}\left(z^{0}\right)\right)\left(w^{0}-h_{2}\left(z^{0}\right)\right)
$$

because $m$ represents $q$. Thus $w^{0}=h_{j}\left(z^{0}\right)$ for some $j$ and $q \in V_{j}$.

(ii) $V$ is a part of $M(A)$.

It is clear that no point in $V$ is equivalent to any point in $X \backslash V$ as the function $z$ has modulus one on $X \backslash V$. And it is seen directly that any two points in $V_{j}$ are equivalent. Let $p_{j}=\left(0, h_{j}(0)\right) \in V_{j}$. The proof will be complete when we show $p_{1} \sim p_{2}$.

Notice that for $f \in A$ we have

$$
f\left(p_{j}\right)=\frac{1}{2 \pi} \int_{0}^{2 \pi} f\left(e^{i \theta}, h_{j}\left(e^{i \theta}\right)\right) d \theta .
$$


Choose an open arc $J$ on $T^{1}$ such that $\left|h_{i}\right| \leqq \frac{1}{2}$ on $J$. Let $f \in A$, $|f| \leqq 1$. Then

$$
\begin{aligned}
\left|f\left(p_{1}\right)-f\left(p_{2}\right)\right| & \leqq \frac{1}{2 \pi} \int_{0}^{2 \pi}\left|f\left(e^{i \theta}, h_{1}\left(e^{i \theta}\right)\right)-f\left(e^{i \theta}, h_{2}\left(e^{i \theta}\right)\right)\right| d \theta \\
& \leqq \frac{1}{2 \pi} \int_{T \backslash J} 2 d \theta+\frac{1}{2 \pi} \int_{J}\left|f\left(e^{i \theta}, h_{1}\left(e^{i \theta}\right)\right)-f\left(e^{i \theta}, h_{2}\left(e^{i \theta}\right)\right)\right| d \theta .
\end{aligned}
$$

Recall that there is a constant $c<2$ such that whenever $\left|z_{1}\right| \leqq \frac{1}{2}$, $\left|z_{2}\right| \leqq \frac{1}{2}$ and $g$ is in the disc algebra $\left|g\left(z_{1}\right)-g\left(z_{2}\right)\right|<c\|g\|$. Now for $e^{i \theta} \in J, w \rightarrow f\left(e^{i \theta}, w\right)$ is a function in the disc algebra of norm at most one. Since $\left|h_{j}\left(e^{i \theta}\right)\right| \leqq \frac{1}{2}$ for $e^{i \theta} \in J$, we therefore have

$$
\left|f\left(e^{i \theta}, h_{1}\left(e^{i \theta}\right)\right)-f\left(e^{i \theta}, h_{2}\left(e^{i \theta}\right)\right)\right|<c
$$

uniformly on $J$. Hence

$$
\left|f\left(p_{1}\right)-f\left(p_{2}\right)\right| \leqq \frac{1}{2 \pi} \int_{T \backslash J} 2 d \theta+\frac{1}{2 \pi} \int_{J} c d \theta=c^{\prime}<2 .
$$

Consequently, $p_{1} \sim p_{2}$ and the proof is complete.

Added in proof. The author has recently improved Theorem 2 as follows.

TheOREM 3. Let $K$ be a topological space. Then $K$ is homeomorphic to some Gleason part if and only if $K$ is completely regular and $\sigma$-compact.

\section{REFERENCE}

1. A. Gleason, Function algebras, Seminars on Analytic Functions, Institute for Advanced Study, Princeton, N. J., 1957.

UNIVERSITY OF WASHINGTON 